\title{
Morphologic Features of Magnetic Resonance Imaging as a Surrogate of Capsular Contracture in Breast Cancer Patients With Implant-based Reconstructions
}

\author{
Neelam Tyagi, PhD $^{\star}$, Elizabeth Sutton, MD $^{\dagger}$, Margie Hunt, MS $^{\star}$, Jing Zhang, $\mathbf{M S}^{\star}$, Jung Hun \\ Oh, PhD*, Aditya Apte, PhD*, James Mechalakos, PhD*, Molly Wilgucki, MBS $\ddagger$, Emily Gelb, \\ BS $\ddagger$, Babak Mehrara, MD§, Evan Matros, MD§, and Alice Ho, MD $\ddagger$ \\ *Department of Medical Physics, Memorial Sloan Kettering Cancer Center, New York, New York \\ tDepartment of Radiology, Memorial Sloan Kettering Cancer Center, New York, New York \\ ‡Department of Radiation Oncology, Memorial Sloan Kettering Cancer Center, New York, New \\ York \\ §Department of Surgery, Memorial Sloan Kettering Cancer Center, New York, New York
}

\begin{abstract}
Purpose-Capsular contracture (CC) is a serious complication in patients receiving implantbased reconstruction for breast cancer. Currently, no objective methods are available for assessing CC. The goal of the present study was to identify image-based surrogates of CC using magnetic resonance imaging (MRI).
\end{abstract}

\begin{abstract}
Methods and Materials-We analyzed a retrospective data set of 50 patients who had undergone both a diagnostic MRI scan and a plastic surgeon's evaluation of the CC score (Baker's score) within a 6-month period after mastectomy and reconstructive surgery. The MRI scans were assessed for morphologic shape features of the implant and histogram features of the pectoralis muscle. The shape features, such as roundness, eccentricity, solidity, extent, and ratio length for the implant, were compared with the Baker score. For the pectoralis muscle, the muscle width and median, skewness, and kurtosis of the intensity were compared with the Baker score. Univariate analysis (UVA) using a Wilcoxon rank-sum test and multivariate analysis with the least absolute shrinkage and selection operator logistic regression was performed to determine significant differences in these features between the patient groups categorized according to their Baker's scores.
\end{abstract}

Results-UVA showed statistically significant differences between grade 1 and grade $\geq 2$ for morphologic shape features and histogram features, except for volume and skewness. Only eccentricity, ratio length, and volume were borderline significant in differentiating grade $\mathcal{s}$ and grade $\geq 3$. Features with $P<.1$ on UVA were used in the multivariate least absolute shrinkage and

Reprint requests to: Neelam Tyagi, PhD, Department of Medical Physics, Memorial Sloan Kettering Cancer Center, 1275 York Ave, New York, NY 10065. Tel: (212) 639-2957; tyagin@mskcc.org.

Conflict of interest: none.

Supplementary material for this article can be found at www.redjournal.org. 
selection operator logistic regression analysis. Multivariate analysis showed a good level of predictive power for grade 1 versus grade $\geq 2 \mathrm{CC}$ (area under the receiver operating characteristic curve 0.78 , sensitivity 0.78 , and specificity 0.82 ) and for grade $\mathcal{2}$ versus grade $\geq 3 \mathrm{CC}$ (area under the receiver operating characteristic curve 0.75 , sensitivity 0.75 , and specificity 0.79 ).

Conclusions-The morphologic shape features described on MR images were associated with the severity of CC. MRI has the potential to further improve the diagnostic ability of the Baker score in breast cancer patients who undergo implant reconstruction.

\section{Introduction}

Although implant reconstruction provides significant psychological and cosmetic benefits for breast cancer patients, capsular contracture (CC) is a common complication that can be a major source of morbidity (1). The capsules that form around implants are generally thin and consist of woven collagen fibers (2). However, in some patients, abnormal thickening of the collagen fibers with a more parallel orientation results in tightening of the capsule around the implant, leading to symptomatic "capsular contracture" $(3,4)$. Women with significant CC complain of pain, a decreased range of motion, decreased symmetry, poor cosmesis, and impaired quality of life. Factors such as radiation, subclinical infections, hematoma, and the absence of antibiotics during surgery have been proposed as etiologies of CC. However, the contribution of each of these factors to the development of CC is unknown $(1,5,6)$. It has been estimated that up to $30 \%$ of patients will develop CC when undergoing implant reconstruction after mastectomy. Radiation therapy further increases the risk of CC in patients who have undergone implant reconstruction. Studies have indicated that radiation is an independent risk factor for complications in patients with implant reconstruction, including a two- to threefold increase in the risk of significant CC $(7,8)$. Therefore, developing novel methods to reliably and objectively assess and diagnose the rates of $\mathrm{CC}$ is an important clinical goal.

To date, the standard tool for estimating the severity of CC is the Baker classification system, which consists of a 4-point scale that integrates the appearance, texture, and tenderness of the reconstructed breast $(9,10)$. The $\mathrm{CC}$ grade, based on Baker classification, is defined as follows:

Grade 1: The breast appears absolutely natural; no one can discern that the breast was augmented.

Grade 2: Minimal contracture; the surgeon can discern that surgery was performed, but the patient has no complaint.

Grade 3: The breast is firm and is beginning to appear distorted in shape.

Grade 4: Severe contracture is present; on observation, it is obvious that the breast has been augmented.

The Baker system is highly subjective and not readily quantifiable, resulting in high interrater (and possible intrarater) variability $(2,11,12)$. In addition, this classification scheme does not provide any quantitative information regarding changes in implant shape or morphologic changes in the pectoralis muscle covering the implant. Thus, although the 
Baker classification is commonly reported, its utility has not been validated in wellcontrolled studies. This shortcoming is likely a major contributor to the significant variability in the published data on the rates of CC.

Magnetic resonance imaging (MRI) has become a standard diagnostic imaging modality to assess implant integrity. However, few studies have correlated the CC outcomes using MRI and clinical evaluations (13-15). A qualitative visual evaluation of the MR images demonstrated a more rounded implant for patients with Baker grade 3 to 4 compared with grade 1 CC (Fig. E1; available online at www.redjournal.org). This has also been the clinical observation or experience of physicians. A clinical case demonstrating that the shape of the implant becomes more rounded and rises more superiorly on the chest wall when the patient develops severe grade $3 \mathrm{CC}$ compared with grade $1 \mathrm{CC}$ is shown in Figure 1. The best method to mathematically represent the implant shape is by calculating the morphologic shape features. It is also known that surgery and radiation can result in fibrosis of the nearby tissue and muscle around the implant. Thus, we hypothesized that either the capsule around the implant or the fibrosis in the muscle near the implant could contribute to CC after implant reconstruction and/or radiation therapy (RT). These observations led us to investigate both the shape features of the implant and the histogram features of pectoralis muscles to quantitatively and objectively assess CC.

The purpose of the present study was therefore to identify quantitative and morphologically based surrogates of CC using MRI. This is a feasibility study to find imaging features in a population of breast cancer patients with permanent implant reconstruction (with and without CC) who had undergone MRI within months of their clinical assessment of CC by a plastic surgeon.

\section{Methods and Materials}

\section{Patient data}

After institutional review board approval, all patients with nonmetastatic breast cancer at diagnosis who had undergone prosthesis-based reconstruction, with or without RT to the temporary expander or permanent implant from January 2008 to 2014 at our institution, were identified. Of these patients, 50 met our study criteria of having a diagnostic MRI performed within 6 months of a plastic surgeon's evaluation of the CC score during postoperative follow-up. All patients had undergone 2-stage tissue expander or permanent implant breast reconstruction by 1 of 6 plastic surgeons specializing in the care of breast cancer patients. As is standard practice, the patients were followed up by the plastic surgeon on an annual basis after permanent implant exchange. The MRI studies performed on these patients were acquired as a part of standard care to evaluate breast integrity, asymmetry, and implant rupture or leakage. The chronological order of surgery, RT, MRI, and Baker's classification is shown in Figure 2. The median time and range for CC, MRI, and Baker's grade were calculated from the time of surgery. Additional patient characteristics by CC grade for the present study are listed in Table E1 (available online at www.redjournal.org). Of the 50 patients investigated in the present study, $17(34 \%)$ had grade 1, $14(28 \%)$ had grade 2, and $19(38 \%)$ had grade 3 or $4 \mathrm{CC}$. The average interval between the surgeons' grading using the Baker classification and the MRI procedure was 2.2 months. Of the 17 
patients with grade $1 \mathrm{CC}, 14$ with grade $2 \mathrm{CC}$, and 19 with grade $3 \mathrm{CC}, 3(17.6 \%), 12$ (85.7\%), and 19 (100\%), respectively, had received postmastectomy radiation therapy (PMRT). The median time from the end of surgery to MRI measurement was 43.0 months (range 16.4-92.6), 43.5 months (range 13.5-102), and 43.8 months (range 34.3-127.5) for grade 1 , grade 2 , and grade 3 to $4 \mathrm{CC}$, respectively.

\section{Imaging details}

All MRI scans were performed using a 1.5 Tesla GE scanner (General Electric Medical Systems, Milwaukee, WI). The scans were acquired using an 8-channel prone surface breast coil. The implant protocol consisted of sagittal $\mathrm{T}_{2}$-weighted (T2W), 2-dimensional fast spin echo (repetition time [TR]/excitation time [TE] 3500/102 ms, slice thickness $4 \mathrm{~mm}$, gap 1 $\mathrm{mm}$ ), axial inversion recovery water-saturated (TR/TE 5000/34 ms, slice thickness $5 \mathrm{~mm}$, gap $1 \mathrm{~mm}$ ), and axial T2W 2-dimensional fast spin echo fat-saturated silicone suppressed (TR/TE 5000/120 ms, slice thickness $5 \mathrm{~mm}$, gap $1 \mathrm{~mm}$ ) scans. Only the sagittal T2W series were used in the present analysis, which included a qualitative, visual evaluation of the images and quantitative contouring of the implant and pectoralis major muscle, with subsequent analysis of the morphologic shape and imaging features.

\section{Image analysis and image features}

The sagittal T2W MR image was imported into the Computation Environment for Radiotherapy Research (CERR) package for analysis (16). The implant shape was segmented on all MRI slices (16). The pectoralis muscle near the implant was segmented on only the 2 central slices, where the muscle was separated owing to the implant insertion. A 2 -cm length of pectoralis muscle, from the top of the implant, was drawn on these 2 slices. The morphologic shape features and first-order histogram features were analyzed according to these regions of interest to investigate whether statistically significant differences were present between the Baker grades for CC (17). MATLAB (MathWorks, Natick, MA) was used to compute the area, perimeter, major axis length, minor axis length, roundness or circularity ratio, eccentricity, solidity, extent, and ratio length within the implant. These shape features were calculated for each slice and then averaged across all the slices within the regions of interest.

1. Roundness was defined as a shape's similarity to a circle and calculated as

$$
\text { Roundness }=\frac{4 \pi * \text { Area }}{(\text { Perimeter })^{2}}
$$

2. Eccentricity was defined as a measure of deviation of an elliptical path from a perfect circle and is a measure of the aspect ratio. It was calculated as the ratio of the length of the bounding box (the smallest rectangle containing every point in the shape) with respect to its width.

3. Solidity is a measure of convexity and describes the extent to which a shape is convex or concave. It was calculated as the ratio of the area of the shape region with respect to the convex hull area of the shape. The convex hull is defined as 
the smallest convex polygon that can contain the region. Some of these shape features are depicted in Figure E2 (available online at www.redjournal.org).

4. Extent is similar to solidity and was defined as the ratio of the area of the object with respect to the surrounding rectangular bounding box. Solidity is a more sensitive measure of a shape with protrusions or an irregular shape than extent.

5. Ratio length was defined as the ratio of the major and minor axis length of the implant.

In addition to the morphologic shape features, the volume of the implant and first-order histogram features were calculated. For the pectoralis muscle, the thickness and first-order histogram features of the MRI signal intensity were calculated. The thickness was measured in the pectoralis muscle that lay immediately caudal to the superior aspect of the implant. The histogram features included the median, kurtosis, and skewness of the pectoralis muscle. Skewness represents a measure of asymmetry of the data around the sample mean. Kurtosis is a measure of how outlier-prone a distribution is. The capsule thickness around the implant on the T2W sagittal MR images was also calculated by measuring the thickness of the thin dark band on the MR images at 4 points around the implant on the central-most image slice (Fig. 3). An average of these 4 measurements was considered the capsular thickness.

\section{Statistical analysis}

Univariate analysis (UVA) using a Wilcoxon rank-sum test was performed to find statistically significant differences in the morphologic shape features of the implant and histogram features of the pectoralis muscle among the patient groups categorized according to the severity of CC (Baker grade). The Wilcoxon rank-sum test is a nonparametric approach that uses magnitude-based ranks to test whether a statistically significant difference exists between 2 sample groups. The test also compares the median between the 2 sample groups. In our case, the statistically significant difference was tested for various features for grade 1 versus grade $\geq 2$ and grade $\underline{2}$ versus grade $\geq 3$, separately. For each feature, if the ranks or medians between the 2 groups were significantly separated, the test statistic identified a statistically significant difference. Receiver operating characteristic (ROC) curve analysis was performed for all the features for grade 1 versus grade $\geq 2$ and grade $\mathcal{2}$ versus grade $\geq 3$ to determine the optimal cutoff values using Youden's index. As performance metrics, in addition to the area under the ROC curve (AUC), the sensitivity and specificity for each variable were calculated at its best cutoff.

Features with $P<.1$ on UVA were used in the multivariate analsysis (MVA) without multiple testing corrections. This was done to evaluate as many potential features as possible with MVA, because the number of features decreased to 3 after applying Bonferroni's correction. To remove highly correlated features, Pearson correlation test was conducted. Among the highly correlated features (Pearson correlation coefficient $>0.85$ ), a feature with the lowest $P$ value on UVA was chosen, removing the others. The remaining features were then used in the MVA, performed using the least absolute shrinkage and selection operator (LASSO) logistic regression for grade 1 versus grade $\geq 2$ and grade $\_$versus grade $\geq 3$, separately. The model building process was conducted using a bootstrapped data set, generated with 
replacement, and out-of-bag samples (not included in the bootstrapped data set) were tested on the model. We iterated this process 100 times, and the performance metrics were averaged.

For the statistical analysis, R language (version 3.2.4) and MATLAB (version 8.6.0) were used.

\section{Results}

\section{Quantitative evaluation of morphologic and MRI features}

CC thickness-The variation in CC measured on the T2W sagittal image is shown in Figure 3. Our measurement showed that the thickness of the capsule was quite similar between those groups with different CC grades, and the difference was not statistically significant.

Implant shape-The results of the analysis of the morphologic features and the relationship to Baker's grade are summarized in Figure 4. The variability in the shape and volume features are represented by a boxplot, with the box representing the 25th and 75th percentiles and the horizontal line, the median value. Implant volume was not a significant feature in differentiating among any grade of CC. Among the morphologic shape features, roundness, solidity, and extent increased with Baker's grade, and eccentricity and ratio length decreased with Baker's grade. The shape feature roundness was statistically significant in differentiating between grades 1 and $2(P=.009)$ and between grades 1 and 3 to $4 \mathrm{CC}(P=.001)$. Eccentricity was borderline significant in differentiating between grades 1 and $2(P=.06)$ but was statistically significant in differentiating between grades 1 and 3 to 4 ( $P=.006)$. Neither roundness nor eccentricity could differentiate grade 2 from grade 3 to 4 CC $(P=.23$ and $P=.12$, respectively). Solidity and extent were statistically significant in differentiating between grades 1 and 3 ( $P=.04$ and $P=.04$, respectively) and marginally significant in differentiating between grades 1 and 2 ( $P=.09$ and $P=.07$, respectively) but not statistically significant in differentiating between grades 2 and 3 to $4 \mathrm{CC}(P=.8$ and $P=.65$, respectively). The ratio length was statistically significant in differentiating between grades 1 and $2(P=.01)$ and grades 1 and $3(P=.001)$ and borderline significant in differentiating between grades 2 and 3 to $4 \mathrm{CC}(P=.06)$. The first-order histogram features (mean, median, kurtosis, and skewness of the intensity values inside the implant) did not show any correlation with CC grade. Kurtosis showed a slight decrease and skewness a slight increase from grade 1 to grade 3 to 4 ; however, the trend was not statistically significant.

Pectoralis muscle thickness-Because the pectoralis muscle on a sagittal T2W image is an elongated structure, we did not expect, nor did we observe, the roundness, eccentricity, extent, and solidity features to demonstrate any clear relationship with CC. The pectoralis muscle thickness showed an increasing trend with $\mathrm{CC}$ grade and was statistically significant in distinguishing grade 1 from grade $3 \mathrm{CC}$ ( $P=.02$; Fig. 5$)$. However, it was unable to differentiate grade 1 from grade 2 or grade 2 from grade 3 to 4 CC. Both skewness and kurtosis showed a decreasing trend from low- to high-grade CC; however, again, the trend was not statistically significant, except for kurtosis, which was able to differentiate grade 1 from grade $3 \mathrm{CC}(P=.01)$. The median intensity values of the pectoralis muscle showed a 
slight increasing trend and were statistically significant in differentiating between grade 1 and grade $2 \mathrm{CC}(P=.03)$ but not the higher grades of $\mathrm{CC}$.

\section{ROC curve and MVA}

UVA and MVA were performed in dichotomized groups with 2 scenarios: grade 1 versus grade $\geq 2$ and grade $\mathcal{\imath}$ versus grade $\geq 3$. UVA performed using the Wilcoxon rank-sum test showed statistically significant differences between grade 1 and grade $\geq 2$ for morphologic shape features and histogram features, except for volume and skewness (Table E2; available online at www.redjournal.org). Only eccentricity, ratio length, and volume were borderline significant in differentiating grade $\mathcal{S}$ and grade $\geq 3$. The AUC, sensitivity, and specificity at the best cutoff values for the morphologic shape features and histogram features for grade 1 versus grade $\geq 2$ and grade $\mathcal{2}$ versus grade $\geq 3$ are listed in Table 1 . The ROC curves for all the features for grade 1 versus grade $\geq 2$ are shown in Figure E3 (available online at www.redjournal.org).

Features with $P<.1$ using the Wilcoxon rank-sum test were used in LASSO logistic regression analysis. For grade 1 versus grade $\geq 2,8$ features, except for volume and skewness, were selected. For grade $\mathcal{\Omega}$ versus grade $\geq 3,3$ features were selected, including eccentricity, ratio length, and volume (Table E2; available online at www.redjournal.org).

Pearson correlation test showed that the roundness and ratio length were highly correlated ( $\rho$ $=-0.9$; Figure E4; available online at www.redjournal.org). Because of the greater significance of the ratio length on UVA, roundness was removed, resulting in 7 features in the LASSO logistic regression analysis for grade 1 versus grade $\geq 2$. After 100 iterations of the model-building process, a good level of predictive power was obtained, with an AUC of 0.78 , sensitivity of 0.78 , and specificity of 0.82 for grade 1 versus grade $\geq 2$ and AUC of 0.75 , sensitivity of 0.75 , and specificity of 0.79 for grade $\mathcal{s}$ versus grade $\geq 3$. For the features used in the LASSO logistic regression analysis, the frequency of the occurrence of each feature was counted during 100 iterations of the model-building process. In the models for both grade 1 versus grade $\geq 2$ and grade $\mathcal{\Omega}$ versus grade $\geq 3$, the ratio length was most frequently selected, at 98 and 94 times for grade 1 versus grade $\geq 2$ and grade $\mathcal{2}$ versus grade $>3$, respectively (Fig. E5; available online at www.redjournal.org).

\section{Discussion}

In the present study, we investigated the surrogates of CC using MRI in breast cancer patients who had undergone implant reconstruction after mastectomy. Our data suggest that MRI can provide quantitative metrics to assess the severity of CC. To date, only 1 study has investigated capsular thickness on imaging studies and correlated the findings with the Baker scoring system (14). Zahavi et al (14) reported on 20 patients who had undergone breast reconstruction with implants and were then followed up for potential CC using both MRI and ultrasound to evaluate the radiologic thickness of the capsule. MRI revealed the distinct appearance of a thickened capsule that correlated with the Baker scoring system. A statistically significant proven correlation was found between the capsular thickness on MRI and the clinical Baker score $(P=.02)$. A Baker score of 1 or 2 correlated with a thinner capsule, averaging $1.14 \mathrm{~mm}$, and a Baker score of 3 or 4 correlated with a thicker capsule, 
averaging $2.62 \mathrm{~mm}$ (14). In contrast to these findings, we did not observe any difference in capsule thickness between low- and high-grade CC on the MR images (Fig. 4). In addition to the sample size difference, our measurements did not include the capsular thickness in regions of the implant where a significant overlap was present between the muscle or the surrounding tissue and the capsule. However, we visually observed a distinct difference in the shape of the implant and pectoralis muscles, which led us to investigate the morphologic shape features as a surrogate of CC (18). That investigation demonstrated that a simple measure, such as implant volume, might be insufficient for distinguishing low- from highgrade CC. However, the shape features of roundness, eccentricity, solidity, extent, and ratio length were significant to various degrees in differentiating among low (grade 1), moderate (grade 2), and severe (grade 3-4) grades of CC. The ROC curve analysis results suggested that roundness, eccentricity, and ratio length are the most reliable features for predicting the risk of developing CC (Table 1; Fig. E3; available online at www.redjournal.org). In particular, the greatest AUC for ratio length is consistent with its importance on MVA using LASSO logistic regression analysis with the greatest frequency of occurrence. The Pearson correlation results among these metrics indicated that eccentricity correlated negatively with roundness and positively with the ratio length. These shape features all indicated that the implant shape progressively becomes more rounded as the CC increases. This is consistent with what has been observed clinically when a patient develops $\mathrm{CC}$, in which the shape of the implant becomes more rounded and rises more superiorly on the chest wall (Fig. 1).

Surgery and/or RT can cause fibrosis in the capsule around the implant or in the muscle near the implant, which could contribute to CC. Most patients who developed moderate to severe CC in the present study had undergone PMRT. RT might have enhanced the degree of fibrosis in the capsule around the implant, as well as the fibrosis in the pectoralis muscles overlying the breast implant. RT results in a hardening of this muscle, which could distort the shape of the implant. Our analysis showed that the pectoralis muscle was thicker in patients with moderate to severe CC compared with that in patients with low-grade CC (6.6 $\mathrm{mm}$ vs $8.7 \mathrm{~mm}$ ). This could have more likely resulted from post-RT fibrosis or inflammation. We considered the median, kurtosis, and skewness of the MRI signal intensities. However, skewness was not statistically significant in differentiating the grades of CC. The trends observed for the median and kurtosis might be indicative of RT-induced effects to the muscle, because most (14 of 17) of low-grade patients had not undergone RT, but most of the intermediate- and all the high-grade patients had undergone RT. MRI intensities, as measured in the present study, are not an absolute measure and are dependent on the scanner parameter. A more quantitative measure of the MRI signal by calculating $\mathrm{T} 1 \mathrm{w}$ and $\mathrm{T} 2 \mathrm{w}$ quantitative maps in the pectoralis muscle might provide a more robust and quantitative assessment of the effect of radiation on CC grade and will be a subject of our future work. Such measures are theoretically independent of experimental settings, such as radiofrequency field inhomogeneity, coil sensitivity, and so forth, and might be comparable between patients uniformly scanned on the same scanner (19). The shape features are independent of the MRI intensity, field strength, and manufacturer and are more robust as surrogates of CC.

Our study assumed that no potential deformation or distortion had occurred of the implant itself when scanned using the dedicated prone breast coil. Deformation might be more of an 
issue when patients are scanned in the supine position. However, all patients in the present study were scanned in the prone position. Although our study was a feasibility study to identify the imaging features or surrogates of $\mathrm{CC}$, we were able to identify these features with high sensitivity and specificity using both UVA and MVA, even with a small patient cohort. Future work will incorporate a prospective, large patient cohort study in which both MRI and clinical assessments for CC will be performed synchronously. These surrogates could further increase the diagnostic ability of the Baker score and help to quantify CC more objectively, enabling researchers to more accurately compare the outcomes designed to decrease the rate of this complication and provide patients with better solutions.

\section{Conclusions}

The morphologic shape features described on MR images correlated with the severity of CC in breast implant patients. These features, if validated in a larger prospective cohort of patients with breast reconstruction and undergoing RT, have the potential to be used as a predictor of CC and minimize the subjectivity associated with the current assessment methods.

\section{Acknowledgments}

This research was partially supported by the Memorial Sloan Kettering Cancer Center Support Grant/Core Grant P30 CA008748. N.T. would like to thank Thomas Findley, MD, PhD, Professor of Physical Medicine, New Jersey Medical School, for discussions regarding the potential role of the pectoralis muscle in causing capsular contracture after postmastectomy radiation therapy.

\section{References}

1. Gabriel SE, Woods JE, O'Fallon WM, et al. Complications leading to surgery after breast implantation. N Engl J Med. 1997; 336:677-682. [PubMed: 9041097]

2. Embrey M, Adams EE, Cunningham B, et al. A review of the literature on the etiology of capsular contracture and a pilot study to determine the outcome of capsular contracture interventions. Aesthetic Plast Surg. 1999; 23:197-206. [PubMed: 10384019]

3. Coleman DJ, Sharpe DT, Naylor IL, et al. The role of the contractile fibroblast in the capsules around tissue expanders and implants. Br J Plast Surg. 1993; 46:547-556. [PubMed: 8252260]

4. Piscatelli SJ, Partington M, Hobar C, et al. Breast capsule contracture: Is fibroblast activity associated with severity? Aesthetic Plast Surg. 1994; 18:75-79. [PubMed: 8122581]

5. Rubino C, Mazzarello V, Farace F, et al. Ultrastructural anatomy of contracted capsules around textured implants in augmented breasts. Ann Plast Surg. 2001; 46:95-102. [PubMed: 11216632]

6. Siggelkow W, Faridi A, Spiritus K, et al. Histological analysis of silicone breast implant capsules and correlation with capsular contracture. Biomaterials. 2003; 24:1101-1109. [PubMed: 12504533]

7. McCarthy CM, Pusic AL, Disa JJ, et al. Unilateral postoperative chest wall radiotherapy in bilateral tissue expander/implant reconstruction patients: A prospective outcomes analysis. Plast Reconstr Surg. 2005; 116:1642-1647. [PubMed: 16267426]

8. Whitfield GA, Horan G, Irwin MS, et al. Incidence of severe capsular contracture following implant-based immediate breast reconstruction with or without postoperative chest wall radiotherapy using 40 Gray in 15 fractions. Radiother Oncol. 2009; 90:141-147. [PubMed: 18977547]

9. Spear SL, Baker JL Jr. Classification of capsular contracture after prosthetic breast reconstruction. Plast Reconstr Surg. 1995; 96:1119-1124. [PubMed: 7568488]

10. Mara JE, Baker JJ Jr. Diagnosis and treatment of masses in the augmented breast. Rocky Mt Med J. 1978; 75:255-257. [PubMed: 694338] 
11. O’Toole M, Caskey CI. Imaging spectrum of breast implant complications: Mammography, ultrasound, and magnetic resonance imaging. Semin Ultrasound CT MR. 2000; 21:351-361. [PubMed: 11071616]

12. Rotatori DS, Hathaway CL, Steinbach BG, et al. Noninvasive assessment of implant capsules. Plast Reconstr Surg. 1991; 87:703-708. [PubMed: 2008468]

13. Prantl L, Englbrecht MA, Schoeneich M, et al. Semiquantitative measurements of capsular contracture with elastography-First results in correlation to Baker score. Clin Hemorheol Microcirc. 2014; 58:521-528. [PubMed: 24418866]

14. Zahavi A, Sklair ML, Ad-El DD. Capsular contracture of the breast: Working towards a better classification using clinical and radiologic assessment. Ann Plast Surg. 2006; 57:248-251. [PubMed: 16929187]

15. Rella L, Telegrafo M, Nardone A, et al. MRI evaluation of postmastectomy irradiated breast implants: Prevalence and analysis of complications. Clin Radiol. 2015; 70:948-953. [PubMed: 26050069]

16. Deasy JO, Blanco AI, Clark VH. CERR: A computational environment for radiotherapy research. Med Phys. 2003; 30:979-985. [PubMed: 12773007]

17. Apte A, Veeraraghavan H, Oh J, et al. SU-E-J-253: The radiomics toolbox in the computational environment for radiological research (CERR). Med Phys. 2015; 42:3324.

18. Headon H, Kasem A, Mokbel K. Capsular contracture after breast augmentation: An update for clinical practice. Arch Plast Surg. 2015; 42:532-543. [PubMed: 26430623]

19. Chang KJ, Jara H. Applications of quantitative T1, T2, and proton density to diagnosis. Appl Radiol. 2005; 19:34-42. 


\section{Summary}

Capsular contracture (CC) is a common complication and major source of morbidity in breast cancer patients receiving implant-based reconstruction. In the present study, we looked at the morphologic shape features of the implant on magnetic resonance imaging scans and found that these features were significant to various degrees in differentiating among low, moderate, and severe grades of CC. Once validated in a prospective study, magnetic resonance imaging has the potential to be used as a predictor of the development of CC. 

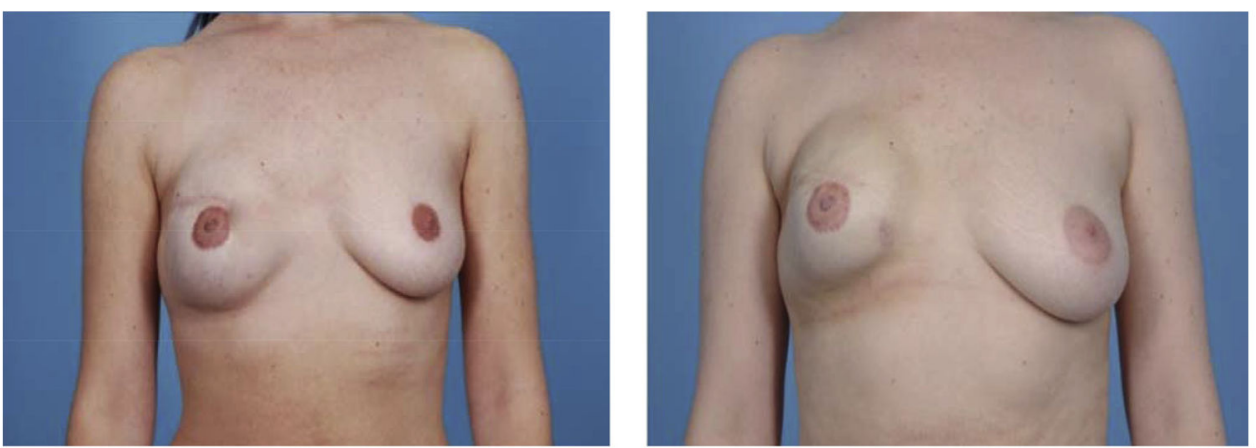

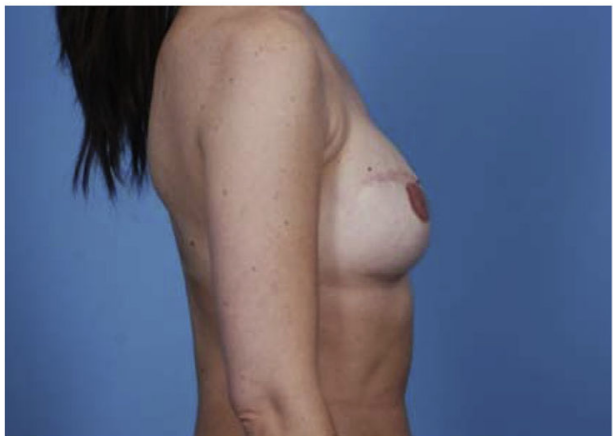

Post mastectomy implant Reconstruction-Grade 1 Capsular contracture

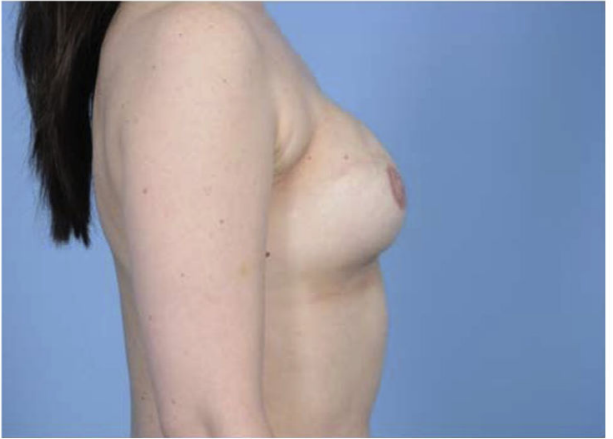

Development of Grade 3 Capsular Contracture

Fig. 1.

Clinical case demonstrating the shape of the implant is more rounded and rising more superiorly on the chest wall when the patient develops severe capsular contracture (grade 3) compared with grade 1 capsular contracture. 
Grade 1

Grade 2

Grade 3-4
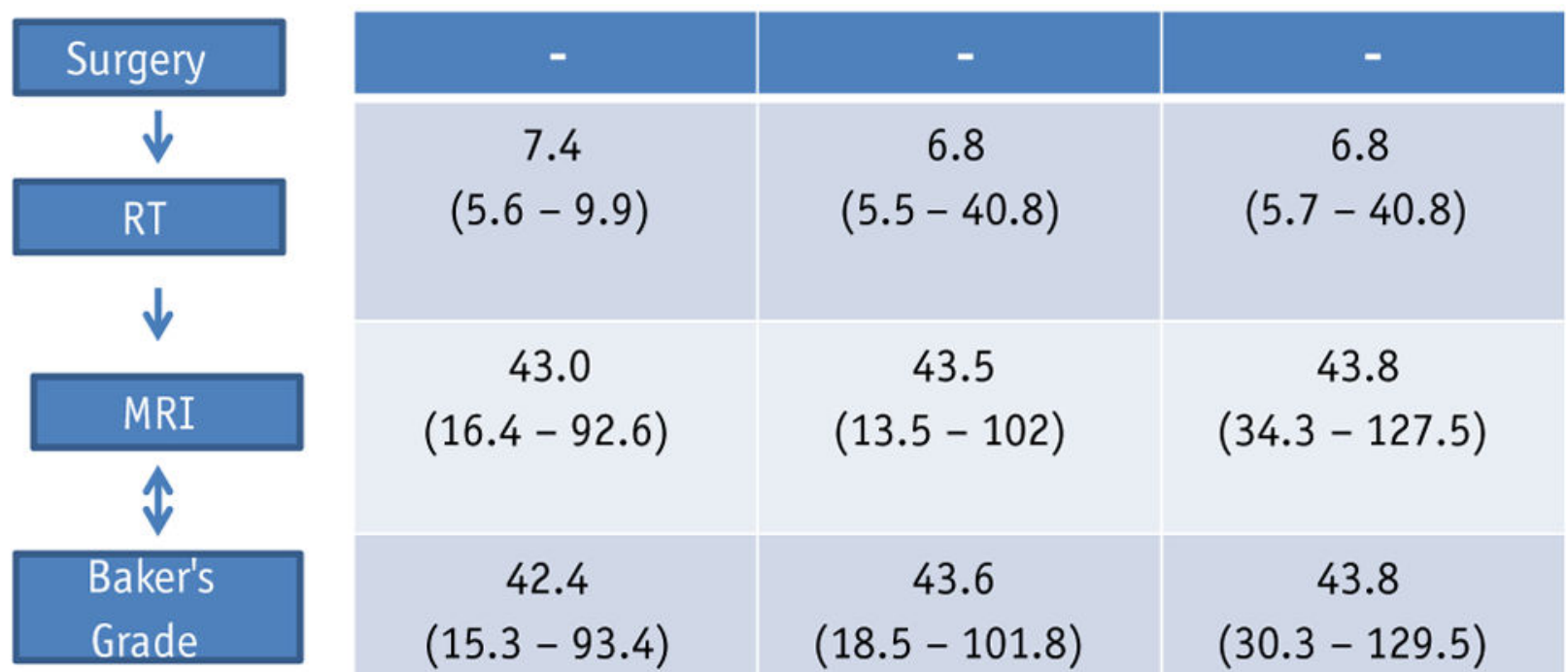

43.0

6.8

6.8

$(5.5-40.8)$

$(5.7-40.8)$

$(16.4-92.6)$

43.5

43.8

MRI

$(13.5-102)$

$(34.3-127.5)$

Grade

42.4

$(15.3-93.4)$
43.6

$(18.5-101.8)$
43.8

$(30.3-129.5)$

Fig. 2.

Chronology of surgery, radiation therapy (RT), magnetic resonance imaging (MRI), and Baker's grade. The time reported for capsular contracture, MRI and Baker's classification was calculated in months from the time of surgery. 

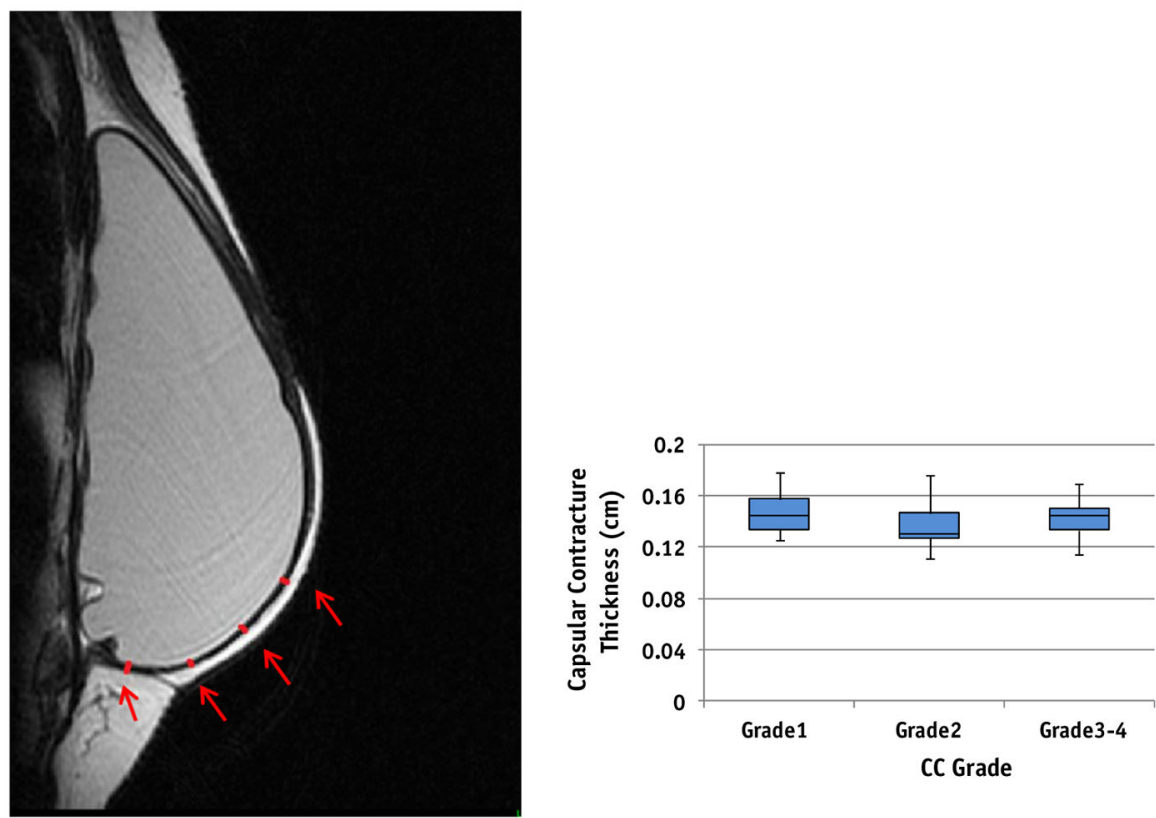

Fig. 3.

Capsular contracture (CC) thickness measured on the T2-weighted sagittal magnetic resonance image. Capsule thickness was calculated at 4 points (red arrows) around the implant on the central-most image slice (left). The box plot (right) shows the capsule thickness calculated as a function of $\mathrm{CC}$ grade. (A color version of this figure is available at www.redjournal.org.) 

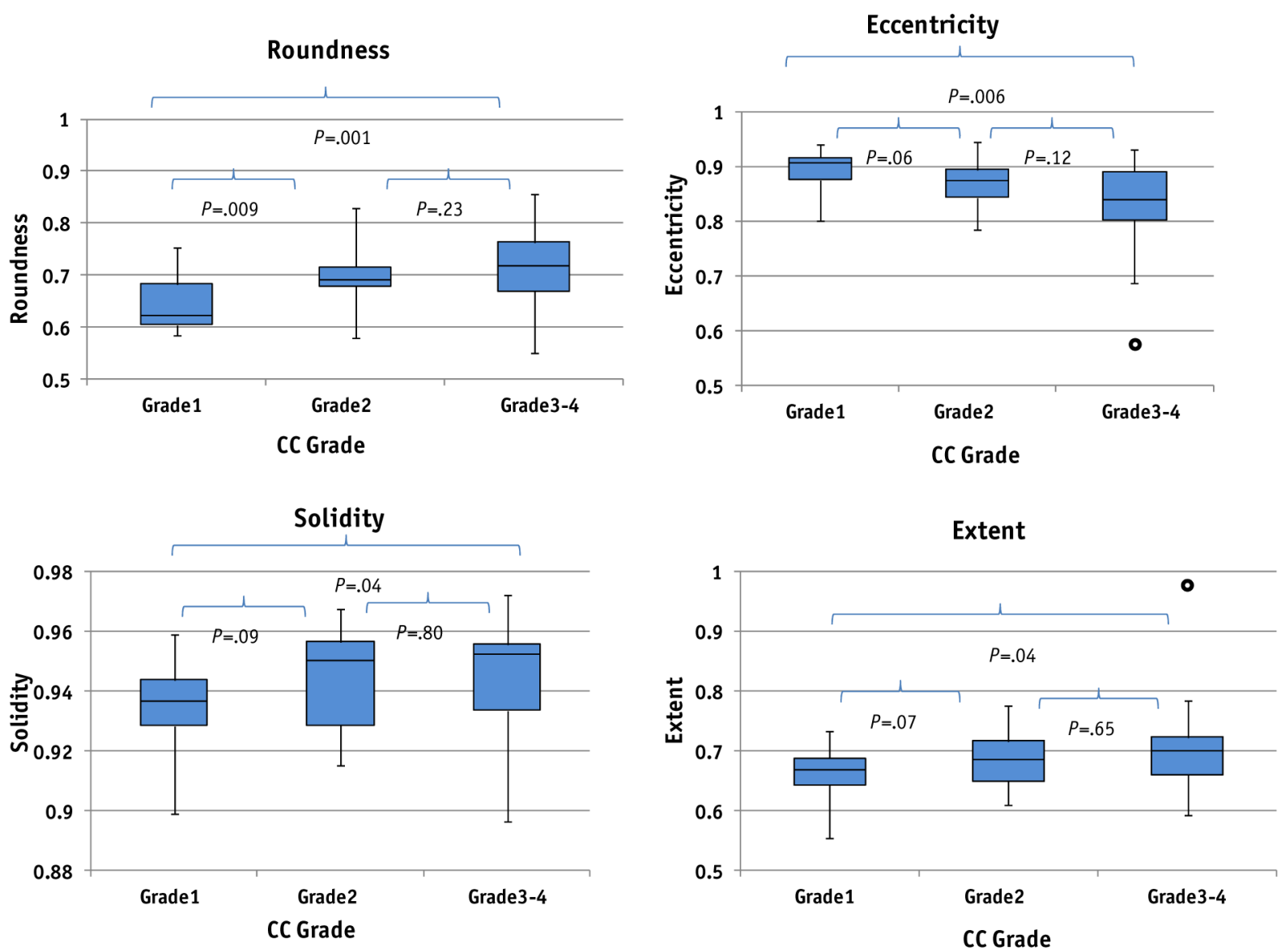

Ratio length
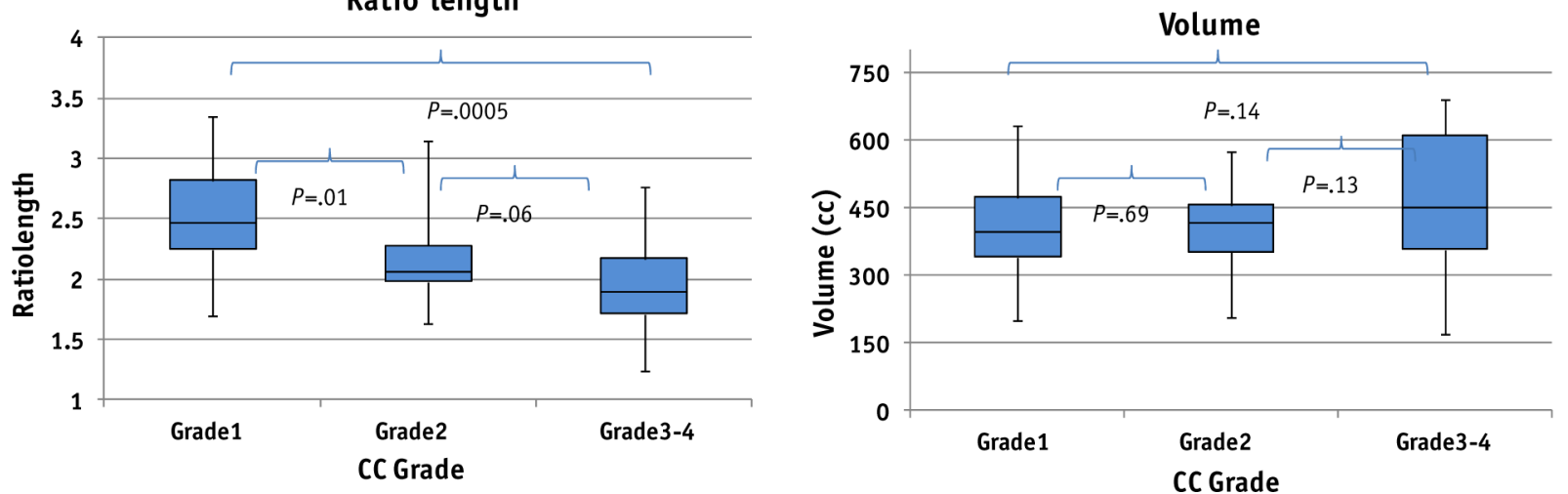

Fig. 4.

Morphologic shape features of roundness, eccentricity, solidity, extent, ratio length, and volume with respect to capsular contracture (CC) grade for the implant. Each shape feature is represented by a boxplot, in which the box represents the 25 th and 75 th percentiles and the horizontal line in the box the median value. 


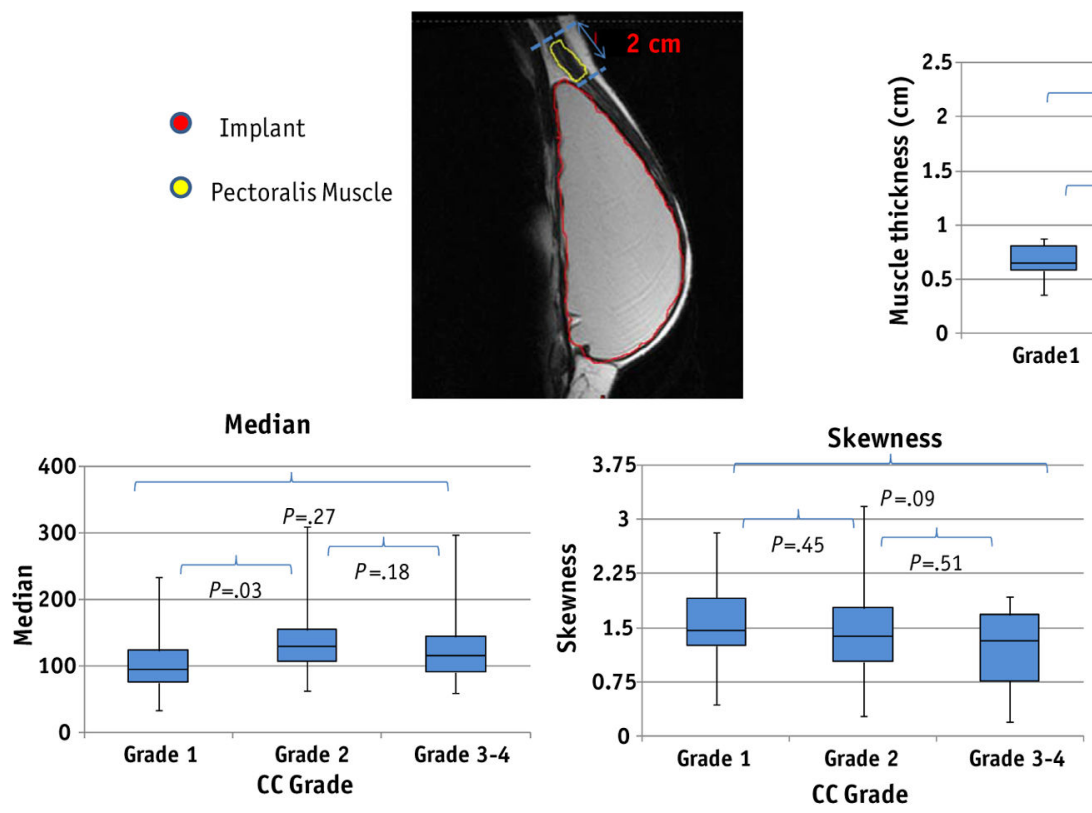

Muscle Thickness

Fig. 5.

Muscle thickness and magnetic resonance imaging intensity histogram features of median, kurtosis, and skewness of volume with respect to the capsular contracture grade for the pectoralis major muscle. Thickness was measured in the pectoralis muscle that lay immediately caudal to the superior aspect of the implant. An attempt was made to contour a consistent $2 \mathrm{~cm}$ of pectoralis muscle on 2 central slices. Each feature is represented by a boxplot, with the box representing the 25 th and 75 th percentiles and the horizontal line in the box the median value. 


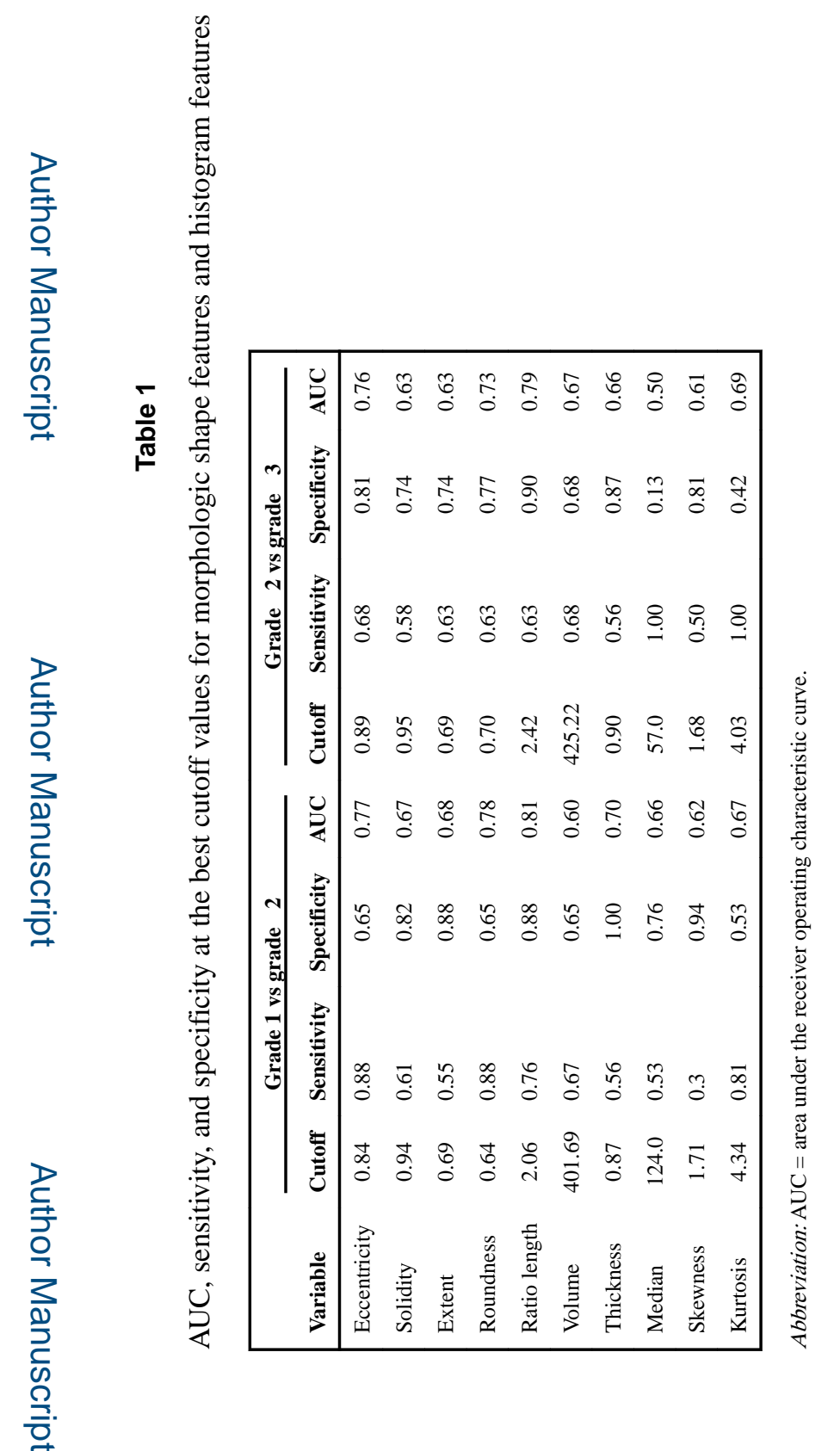

Int J Radiat Oncol Biol Phys. Author manuscript; available in PMC 2018 February 01. 